\title{
The Importance of Representing the Physical Universe or Virtual Universes in Functional State Space to the Progress of Physics
}

Andy E. Williams, Nobeah Foundation, Nairobi, Kenya

\begin{abstract}
:
Human-Centric Functional Modeling is a newly emerging branch of network theory that is hypothesized to define a methodology for representing any system, including the physical universe or any object in it. This paper explores how any region in the physical universe or how any virtual representation of such a region might be represented this way, as well as outlining the benefits of doing so.
\end{abstract}

\section{Introduction}

Following the long tradition in philosophy of defining state spaces as representations of semantic meaning in physics and other disciplines [1], for all systems, the newly emerging science of HumanCentric Functional Modeling or HCFM hypothesizes that the behavior of those systems can be represented in terms of functional state spaces. In a functional state space each region represents a state. Each state is separated from other regions (other states) by the processes through which that state might transition to those other states. In the case that a functional state space is "spanned" by some basic set of functions, these processes represent some combination of those functions. As an example, HCFM represents the functional state space of the human cognitive system in terms of a space of concepts or conceptual space in which the cognition can potentially transition from one concept to another concept through reasoning processes. The importance of being able to apply HCFM to cognition is that it allows the application to any other system to be understood by analogy with this case that is by definition conceptualizable.

This paper describes how HCFM might be used to represent any object in the physical universe, or any object in any virtual universe such as the Metaverse, as well as any physical or virtual universe in their entirety. This paper also proposes a definition for complexity that applies to any functional state space, so that this definition both applies to the space of concepts and reasoning (the conceptual space) that defines our thoughts (concepts and reasoning) about systems, as well as applying to the real or virtual representations of the physical systems themselves. In the case of our thoughts this metric for complexity is hypothesized to measure the complexity of any given concept or reasoning, and in the case of the physical universe this metric for complexity is hypothesized to measure the complexity of any physical transition.

A great many physicists in a great many reputable physics journals (e.g. [2], [3]) have for a long time (since at least the seventies [2]) claimed that network theory related concepts have potentially useful applications in physics, particularly with regards to understanding complexity. As one researcher stated, "if we are ever to have a theory of complexity, it will sit on the shoulders of network theory" [4]. HCFM is merely a particular perspective of network theory. However, this paper does not claim nor to attempt to confirm that the concepts defined by the theory of HCFM are helpful in physics. Instead it attempts to outline some of the questions that must be answered by physicists to determine whether the concepts are helpful to them. The goal of doing so is that if some physicists are able to make the determination that representing the physical or virtual world in terms of functional state space is valid, then with the ability to generalize that might be provided by a General Collective Intelligence or GCI (a hypothetical platform capable of organizing groups to have far greater intelligence than any individual and that depends on information being represented in terms of functional state spaces [7]) it is predicted that the work of those physicists in validating the applicability of functional state space to physics can 
potentially be reused in solving important problems outside physics that are defined in terms of the same networks (functional state spaces).

\section{Representing the Physical Universe as a Functional State Space}

Human-Centric Functional Modeling represents potentially all systems in terms of functional state spaces that are defined by analogy with the functional state spaces that can be used to describe the human system. Because these functional state spaces can be used to describe the human cognitive system, because the behavior of the human cognitive system can by definition be conceptualized, because that conceptualized behavior can be understood in terms of properties of functional state spaces, and because those properties of functional state spaces (like complexity) are potentially applicable to all functional state spaces, then the functional state spaces and properties of functional state spaces (like complexity) defined through Human-Centric Functional Modeling can potentially be used to understand all other systems as well.

Representing the physical universe in terms of functional state spaces is important because it allows physicists to be engaged in solving the remaining problems in elaborating a complete representation of functional state space. Elaborating functional state space is in turn important because there is a strong case that "wicked" problems like poverty and sustainability are patterns in some functional state space [13]. It is hypothesized that understanding these patterns can exponentially increase our capacity to solve these problems to the point that at least some are reliably solvable where that is not the case today.

Consider the functional state space representing a universe consisting of two particles separated by some distance. For each particle there is some moving region in space that represents the physical state of that particle. This region has some uncertainty. The functional state space with which one might compute the evolution of the state of each of the two particles then consists of a region representing each particle and a line representing the forces through which the particles interact.

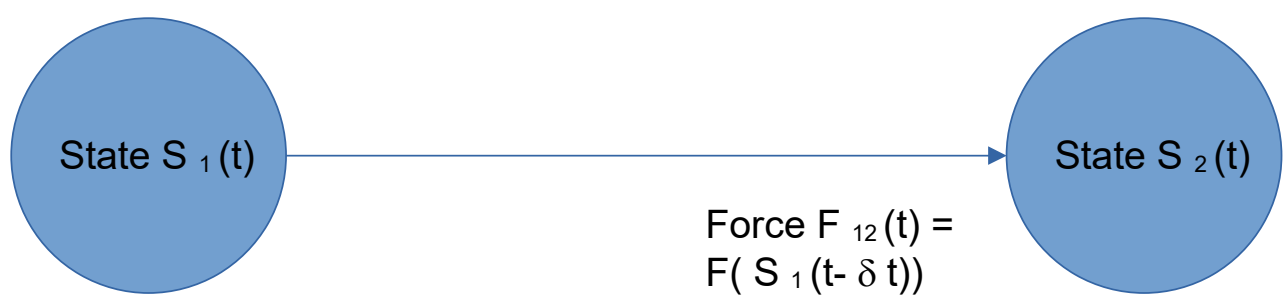

Figure 1: The state of each region is determined by the forces from surrounding regions. The force on region 2 due to region 1 however is the result of the state of region 1 at some earlier time t-dt. The universe is a distributed "cognition" in that it computes the state of every region of matter.

Assume that the functions through which the states of the particles interact are some combination of the four fundamental forces. Assuming force is not propagated instantaneously, the forces at particle 2 at time $t$ are due to the state of particle 1 at some earlier time $t-\delta$ t. Similarly the forces at particle 1 are due to the state of particle 2 at some earlier time. In that this universe of two particles is continually computing how the state of those two particles evolve, it is a distributed state computer. Since this functional state space is simply a representation of matter and not a new model of physics, the state of each particle will evolve according to existing physics, in this case we assume it evolves according to Schrödinger's Equation. 
In addition to the functional state space describing the state of each particle, there is also a functional state space describing the "awareness" of the state of each particle. The awareness of the state of particle 1 travels from particle 1 , and the awareness of the state of particle 2 travels from particle 2 .

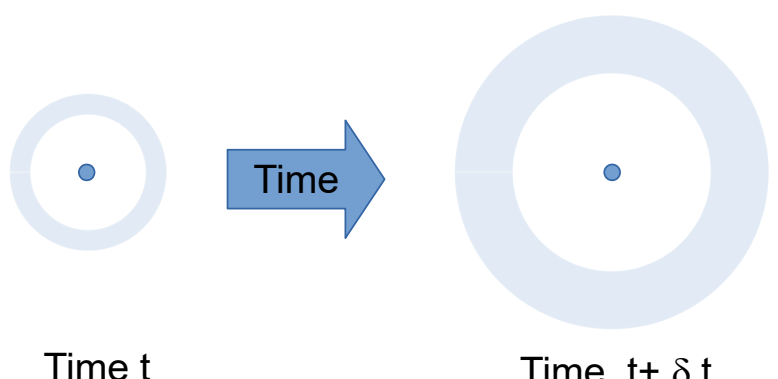

Figure 2: The "awareness" of the state of each region travels in every direction.

Considering each particle alone, assume that the awareness of the state of that particle spreads out in all directions as in figure 2.

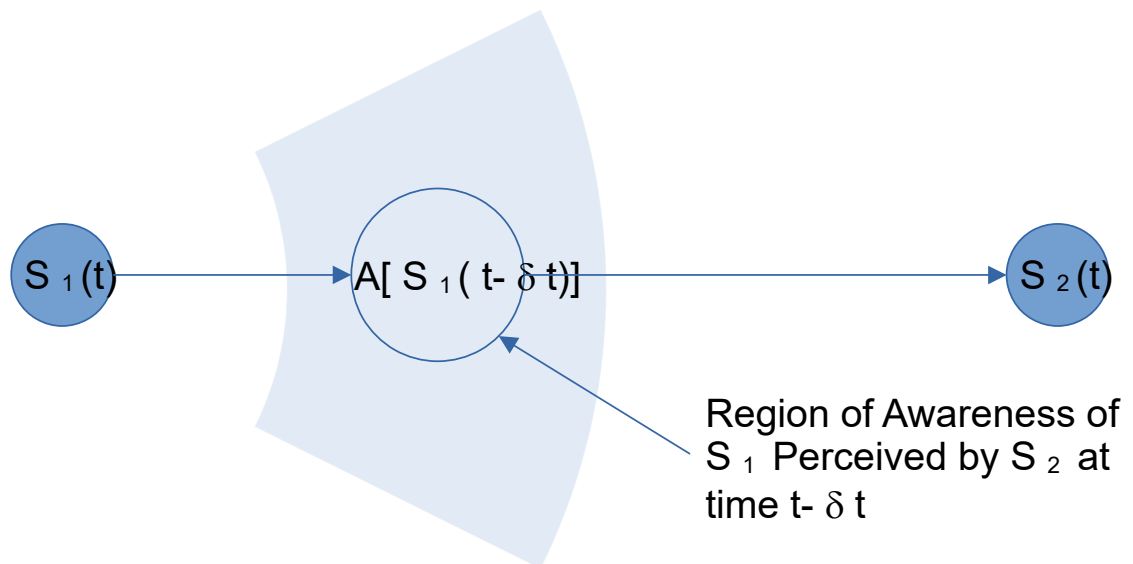

Figure 3: The "awareness" A of the state of particle 1 that is perceived by particle 2 pertains to the state of particle 1 at some earlier time $t$-dt. Furthermore, only a finite region of the awareness of S1 is perceived by $S 2$ at time $t$-dt. Awareness then effectively acts as a network.

The awareness of particle one that is perceived by particle two at any given time occupies a finite region of space that can be represented as a node in a network of awareness's as in figure 4.

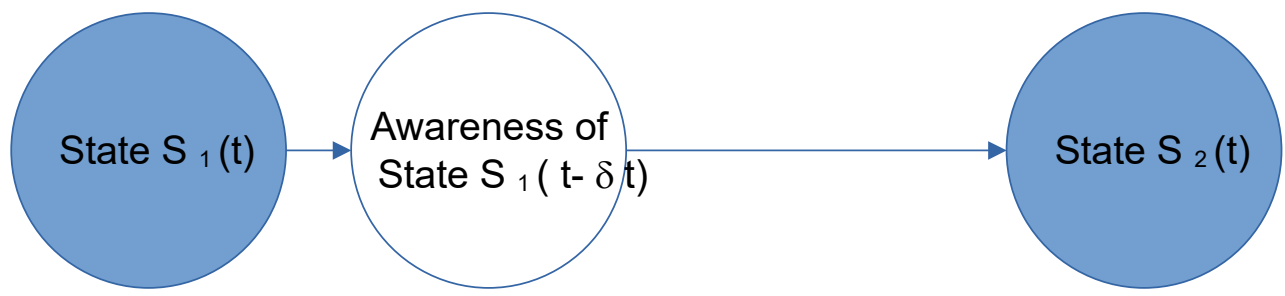

Figure 4: The "awareness" of the state of each region travels through space at some finite velocity. The universe is a distributed "consciousness" in that it is aware of the state of every region of matter. 
Again, since this functional state space is simply a representation of the universe and not a new model of physics, the awareness of state of each particle will evolve according to existing physics, in this case we assume it will evolve according to the Einstein field equations.

Motion through these functional state spaces is not deterministic. Here Human-Centric Functional Modeling contributes to our understanding again. In the model of cognition provided by HumanCentric Functional Modeling, human cognition moves through its functional state space in a nondeterministic way that is dynamically stable in a "fitness space" which describes the fitness of the cognitive system to execute all of its functions. This fitness space is defined in terms of the projected fitness, the target fitness, and the actual fitness of the reasoning function to be executed. The motion of the consciousness through its own functional state space (the awareness space) is also represented as being governed by the requirement for dynamical stability in its own fitness space. Similarly, each particle in this simple universe has a fitness space that describes the fitness of the system. At any time fluctuations due to uncertainty can result in a state that has poor fitness in representing the system. For example, there might be a non-zero probability of a fluctuation in momentum due to uncertainty. With these fluctuations how is momentum still conserved? Our universe must be dynamically stable in that all such fluctuations must remain within stable bounds. Each point in the functional state space of the distributed mind of the universe and each point in the functional state space of the distributed consciousness of the universe might then potentially be represented as evolving under the same algorithm as human cognition in the sense that it must be dynamically stable.

To understand the application to physics, consider a single particle. According to one of potentially other approaches [8], [9] to quantum mechanics, a particle is represented by a wave function that is a solution to Schrodinger's equation [10].

This solution will have some uncertainty in position and momentum. At higher energies the relative uncertainty is less and particles act more like discrete entities. At lower energies the relative uncertainty is greater and particles act more like waves.

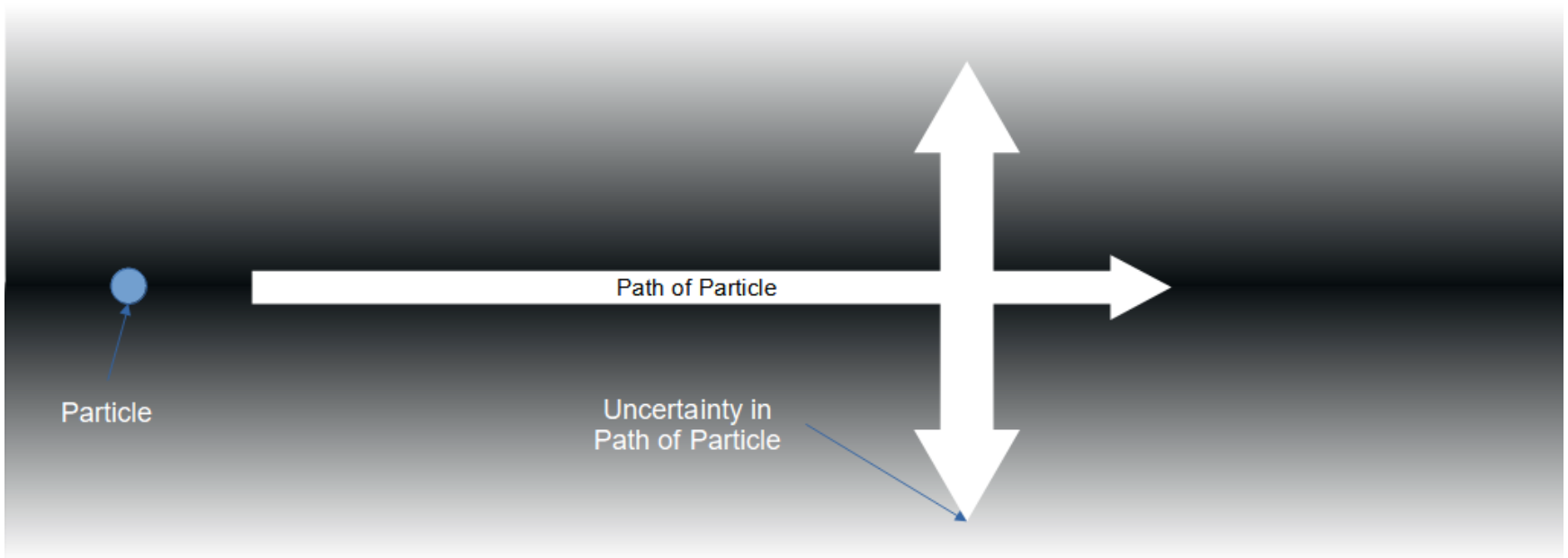

Figure 5: Conceptual representation of the uncertainty in the path of a particle.

When interacting with a field, the wave function must collapse to a specific value for each property with which it interacts. The minimum time interval that is resolvable is a single Planck unit of time. So 
wave functions don't continually collapse, but instead must collapse at those minimum intervals at most. However, due to uncertainty, the value of any property governed by uncertainty, including the time interval at which the wave function collapses, is not deterministic at each time when that wave function collapse might occur.

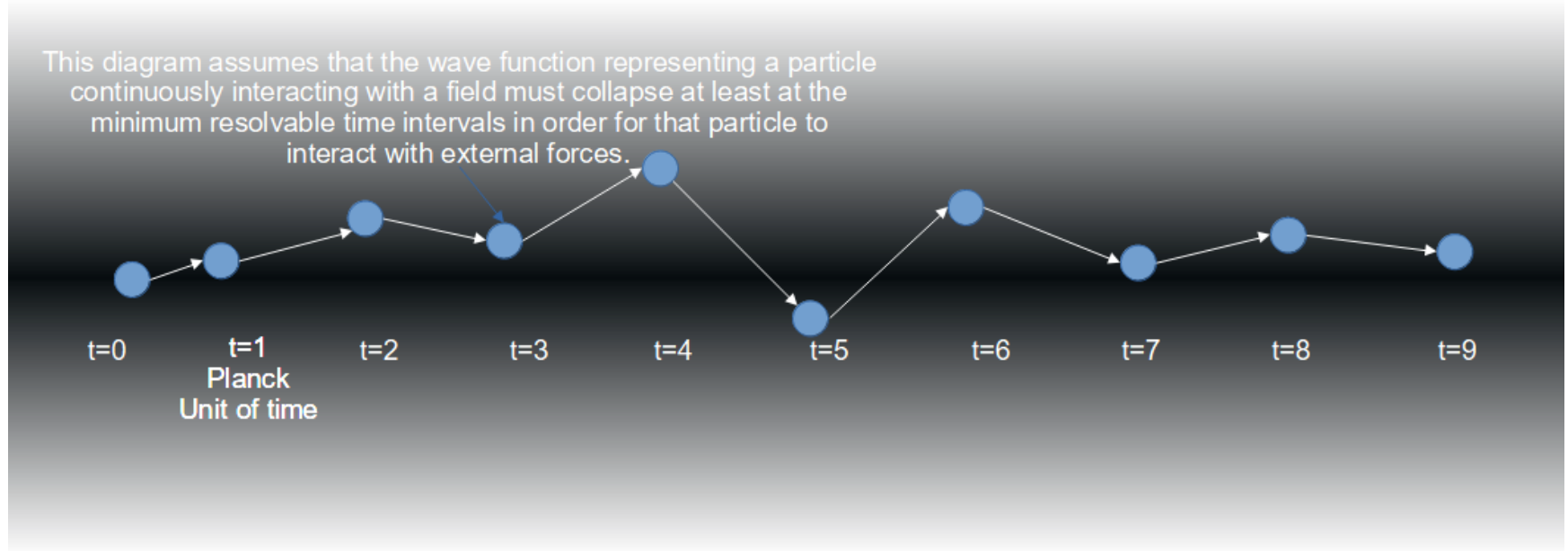

Figure 6: Collapse of the wave function representing a particle continuously interacting with a field.

As a result, any particle might have a target trajectory determined by the current model of physics if it's momentum and other properties are to be conserved from those at $t=0$. It might also have a projected trajectory from its motion and other properties in the last time slice $t=7$. And it might have an actual trajectory at the current time $\mathrm{t}=8$.

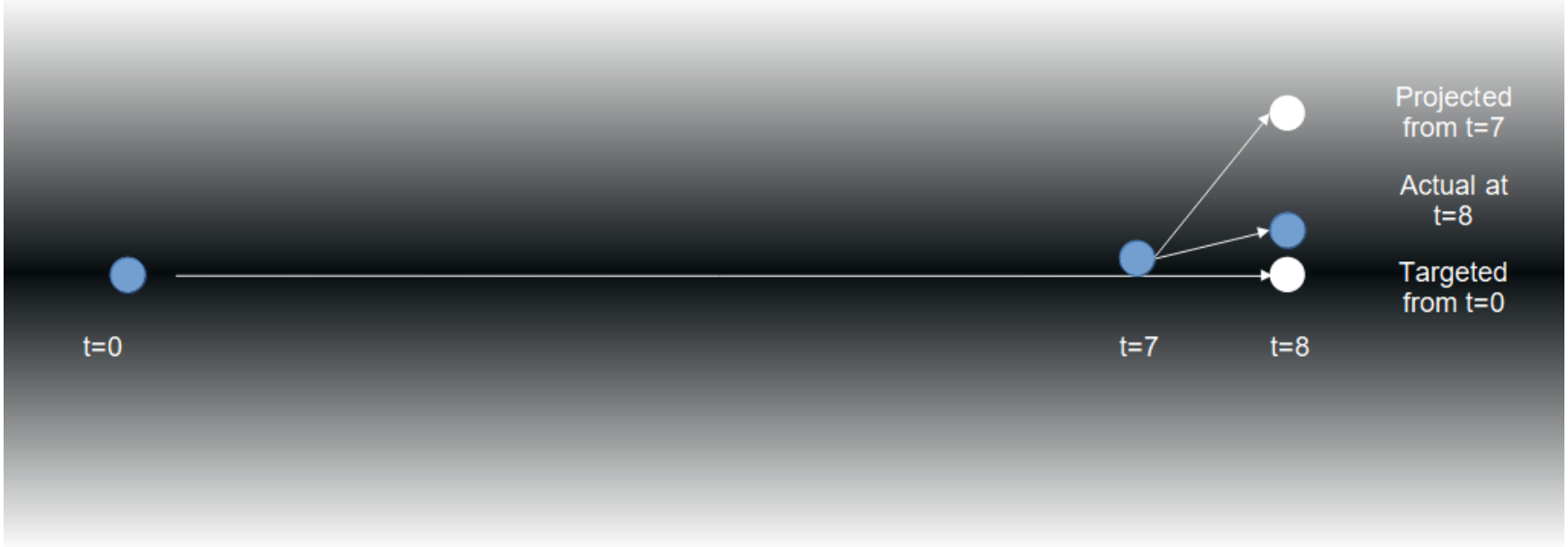

Figure 7: Projected, actual, and targeted positions of the particle from the perspective of HumanCentric Functional Modeling.

In Human-Centric Functional Modeling fitness space is defined by fitness of the projected, actual, and targeted states to represent the particle. The state of the particle must stay in a bounded region in fitness space if momentum and other physical properties are to be conserved. 


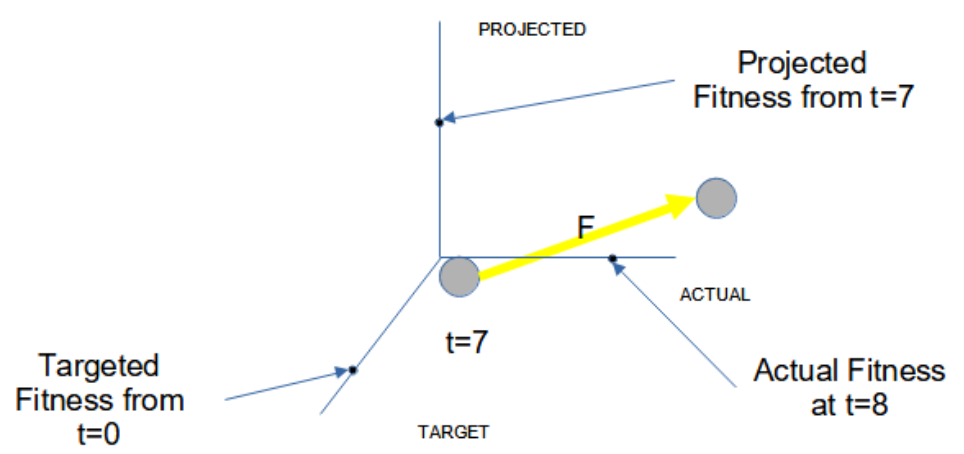

Figure 8: Fitness space of the particle from the perspective of Human-Centric Functional Modeling.

For any theoretical model consisting of a set of theories of physics, any region of matter interacts with any other region of matter through the forces defined by that theoretical model. The key claim of this paper is that there are multiple potential interpretations of the probability distribution described by the hypothetical wave function postulated by quantum mechanics, that each of these interpretations arises from a different philosophy about the nature of this existence, and that each of these philosophies functions to achieve different outcomes, some of which might be more optimal than others in different contexts. The philosophy suggested by Human-Centric Functional Modeling is that many properties of systems are actually properties of the functional state spaces that describe them, and that for all functional state spaces these properties are potentially defined the same way.

By analogy with a particle having a probability of entering a given state after a particular interaction, one might view human cognition as having a particular probability of entering a given state after interacting with a set of concepts and reasoning. Our cognitive system might even be confirmed through experimental observation to have this well defined probability. However, through experiments involving first person observation of our own cognition, we can confirm that the reasoning output by our cognition doesn't just depend on probability and what concept we are currently contemplating (that is, where we are in conceptual space). It also depends on our current state of cognitive well-being (cognitive fitness) in a way which maintains that fitness within a stable region so that our ability to function cognitively is conserved.

Similarly, it is postulated here that for any set of theories of physics that are complete, if properties are to be conserved or other "laws" within those theories are to be upheld, then from the perspective of Human-Centric Functional Modeling that set of theories of physics must keep the state of any given region of matter within a bounded region of some as yet undefined fitness space that describes the fitness of those theories to in turn describe the state of that matter. This implies dynamics of that matter that are globally stable throughout this fitness space for all the forces with which matter or energy might interact as in figure 9. This does not imply any new physics. We assume the dynamics of each region of matter through fitness space can be made to match the predictions of probabilistic quantum mechanics or any other set of theories identically. 


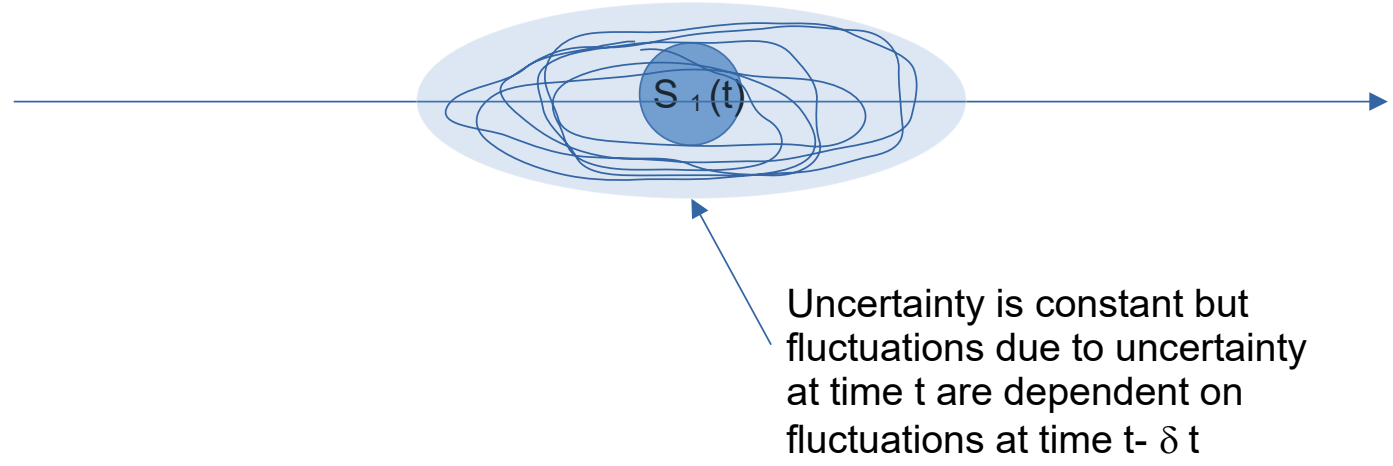

Figure 9: A particle (blue circle) moving in the direction of the horizontal blue arrow has a state $S_{1}(t)$. This particle moves within a cloud of uncertainty around it (light blue oval). If the model of "fitness space" used in defining a model of biological systems such as consciousness or cognition applies to defining a model of physical space, there is potentially structure in the uncertainty in a particles position and momentum in that fluctuations due to uncertainty at one time are correlated with fluctuations at other times. This structure or correlation potentially enables position and momentum to be conserved.

One set of equations that potentially satisfies this stability in the three dimensions of fitness space in order to allow properties to be conserved over time despite fluctuations due to uncertainty are the Lorenz equations governing convection [11], which for certain values of their parameters form a strange attractor.

$$
\begin{aligned}
& \frac{d x}{d t}=\sigma(y-x) \\
& \frac{d y}{d t}=x(\rho-z)-y \\
& \frac{d z}{d t}=x y-\beta z
\end{aligned}
$$

The relationship between the dynamics in functional state space and the dynamics in fitness space in the case of the cognitive system has been hypothesized as one that might leverage such an equation to define an algorithm able to replicate these dynamics in an artificial cognition [12]. The relationship in the case of the physical universe is yet to be explored.

In summary, from the perspective of Human-Centric Functional Modeling this universe can potentially be represented in terms of two functional state spaces, one representing state that is used to determine how the "mind" of the universe calculates the evolution of matter, and one representing awareness of state that is used to determine how the "consciousness" of the universe calculates the evolution of the awareness of the state of matter. 

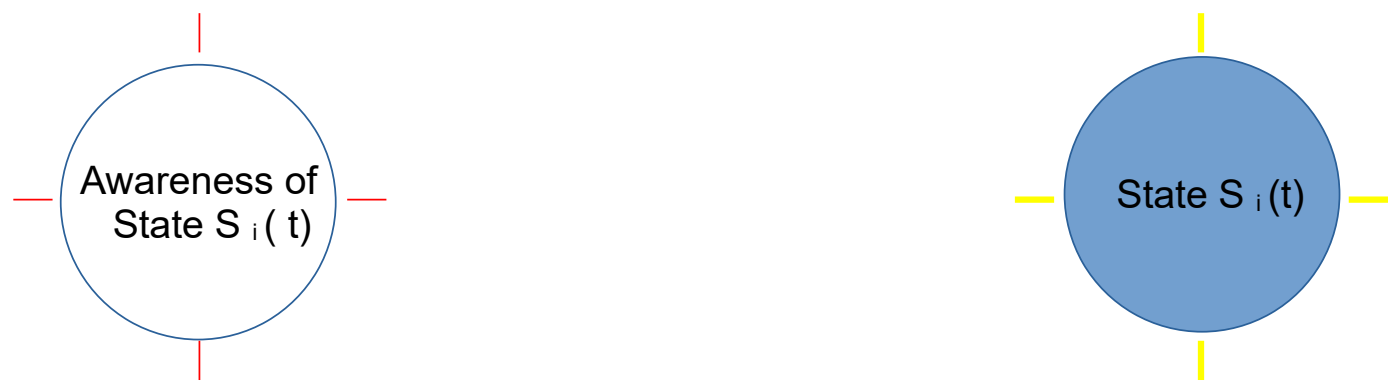

Figure 9: Each region in the functional state space of the "consciousness" of the universe is an awareness of state (left) that interacts with other awareness's through awareness processes indicated by the red lines. Each region in the functional state space of the "mind" of the universe is a state of matter (right) that interacts with other matter through forces indicated by the yellow lines.

\section{Complexity}

In Human-Centric Functional Modeling complexity is hypothesized to be a property of the functional state space describing the behavior of the system. This property is defined as the product of the distance in functional state space between function functional states, multiplied by the linear density of functional states along the transition process which provides the path between those states. Since problems are defined as the lack of a path through functional state space, and since solutions are defined as the processes which provide that path, then any number of solutions might solve a given problem. Since each solution can have a different metric for complexity, this paper proposes that the complexity of a problem in functional state space is defined by the complexity of its least complex solution.

\section{Implications}

Human-Centric Functional Modeling or HCFM has been used to define a model for group decisionmaking called "General Collective Intelligence" or GCI that is hypothesized to have the potential to radically increase the general problem-solving ability (intelligence) of the group so that it is exponentially greater than that of any individual [7]. The usefulness of describing the behavior of human cognition in terms of motion through its functional state space (the conceptual space) is that this functional state space can by definition be conceptualized and therefore be understood. The usefulness of modeling human cognition in terms of motion through its own functional state space is that this understanding of the cognitive system can potentially be reused, and if so the same model used to describe an artificial cognition in terms of a pattern of dynamical stability can potentially be used to describe other biological systems including consciousness, which is useful in defining an artificial consciousness.

The usefulness of using Human-Centric Functional Modeling to describe the behavior of the distributed "mind" of the universe in terms of motion through a distributed functional state space describing state, and the usefulness of using HCFM to describe the behavior of the distributed "consciousness" of the universe in terms of motion through a distributed functional state space describing awareness, is that the same algorithms used to implement an artificial cognition and an artificial consciousness can also potentially be used to simulate the evolution of the physical universe. Furthermore, these same algorithms might also be reused in simulating all other living processes, since those processes are also believed to obey the same pattern of dynamical stability. By solving this far more general problem, it is possible to impact a far larger range of problems. 
It remains to be determined how the concept of functional state space relates to existing concepts. For example, in functional state space the complexity of the transition from one physical state to another has one interpretation suggested by the expression for the complexity of the transition from one position in the functional state space of the cognitive system to another. However, in physical systems, complexity is currently accepted to be a measure of the probability of the state vector of the system. As another example, in astrodynamics and celestial dynamics, the orbital state vectors (sometimes state vectors) of an orbit are Cartesian vectors of position that together with their time (epoch) uniquely determine the trajectory of the orbiting body in space [5]. It remains to be determined how state vectors are related to the states in the functional state space representing the state of each region of matter in the universe.

\section{Discussion}

Noting that GCI requires information to be modeled in a universal way, such as in terms of functional state space, and given that the theory of GCI suggests that there are gaps in the collective conceptual space representing problem definitions or solutions that groups cannot be reliably brought to recognize, and given that in addition the theory of GCI suggests that certain well defined properties of group reasoning that are required to solve wicked problems are not present without GCI [13], the claim can be made that for a number of areas in physics, the key problem is not coming up with a better theory. There may already be a great number of theories that have not been collectively evaluated, and there might be a number of legitimate theories that cannot be given their just due diligence and therefore cannot be collectively evaluated given the way that the dynamics of group reasoning operate in the absence of GCI. Therefore instead of a lack of better theories, the greatest problem and opportunity in physics might be not having a way to compare radically different theories in order to reliably converge on a common understanding regarding which theory is better in describing the observed world. In other words, one could come up with an elegant grand unified theory that describes everything, but according to this reasoning it wouldn't matter, since if it is sufficiently complex the theory of GCI suggests the individual who discovers it would not reliably be able to get a critical mass of people to agree with it.

Success in being able to prove or disprove any sufficiently complex theory in physics using functional state spaces and General Collective Intelligence might seem improbable since there are so many challenges remaining in defining a complete semantic representation of information. However, there might actually a far higher likelihood of being able to succeed in doing so than the probability of achieving success currently might be in working alone or working in groups without GCI. As the number of theories and the amount of data that must be tested against those theories increases, the interactions between those theories and data create an explosion of possibilities to consider. As this continues is is conceivable that scientists might be constrained to become increasingly more powerless to make sweeping cross-disciplinary advances without functional state space and without GCI, no matter how brilliant the scientist is.

\section{Conclusions}

Using functional state space to represent concepts and reasoning can potentially provide a complete semantic representation of concepts and reasoning, as well as potentially explaining how defining our cognition as moving through a functional state space can exponentially increase our general problemsolving ability, and therefore our ability to use reasoning to solve any problem in general, including in physics [6]. In addition to creating the possibility of exponentially increasing our ability to collectively navigate our reasoning, defining functional state spaces also creates the possibility of increasing our ability to collectively navigate states of matter in the actual physical world. Increasing our capacity to navigate reasoning means defining semantic models of reasoning so that we can automate processes that test the consistency of every component of every theory in physics against every component of 
every other theory. Increasing our capacity to navigate data means defining a universal semantic model of the physical world that all data about the physical world fits into, so that all components of all theories can be tested against all data collected by anyone about anything. A universal semantic model of the physical world has countless commercial applications, including the fact that it defines an open rather than proprietary data format for every virtual world, whether the Metaverse, or whether the models of any real or imagined physical environment depicted in movies or video games.

But realizing this vastly increased ability to solve problems in physics also has surprising implications. From the perspective of Human-Centric Functional Modeling our cognition is a computer and our consciousness directs our attention to in essence determine which problems we use that computer to solve. Where our level of intelligence is represented as determining the power of this calculator, our level of consciousness is represented as determining our power to direct our attention towards and therefore to recognize truth. If this science of Human-Centric Functional Modeling is successful in exponentially increasing the power of cognition as predicted through either the model of Artificial General Intelligence or through the model of General Collective Intelligence it has been used to derive, and if theories in physics as well as the data to test those theories on can both be modeled semantically, then the knowledge of even the most talented physicist on earth might be as inconsequential as the mathematical ability of the greatest human calculator who ever lived in comparison with even a lowpower computer today that is many generations old. If so, then in such an age of intelligent machines physicists might pay less attention to the myth that the most significant advances in physics are awaiting the next brilliant physicist, and as a consequence aspire less to be brilliant calculators. Instead they might pay more attention to increasing their level of consciousness and as a consequence their ability to recognize truth, and in doing so aspire more to be like yogis.

\section{Acknowledgments}

Deep thanks for many patient discussions to Don N. Page, a theoretical physicist at the University of Alberta focused on quantum cosmology as well as on theoretical gravitational physics, and in addition a former doctoral student of the eminent Professor Stephen Hawking. Though Professor Page disagreed with the usefulness of the functional state space approach in physics, his generous contribution of his own time to explain his reasons why created the robust discussion that resulted in this paper. In addition, though Professor Page did not specifically endorse the physics content of this paper, that too was beneficial since it resulted in the author's efforts to ensure that the article did not discuss any physics that could not be endorsed, but instead focused on describing functional state space as a tool for representing physical objects and potentially the entire physical universe itself as a network with semantic meaning, and it resulted in the author's effort to ensure that the article focused on understanding the implications of doing so.

\section{References}

[1] Van Fraassen, Bas C. "On the extension of Beth's semantics of physical theories." Philosophy of science 37.3 (1970): 325-339.

[2] Schnakenberg, Jürgen. "Network theory of microscopic and macroscopic behavior of master equation systems." Reviews of Modern physics 48.4 (1976): 571.

[3] Bianconi, Ginestra. "Interdisciplinary and physics challenges of network theory." EPL (Europhysics Letters) 111.5 (2015): 56001.

[4] Barabási, Albert-László. "The network takeover." Nature Physics 8.1 (2012): 14-16.

[5] Howard Curtis (2005-01-10). Orbital Mechanics for Engineering Students (PDF). Embry-Riddle Aeronautical University Daytona Beach, Florida: Elsevier. ISBN 0-7506-6169-0.

[6] Williams, A. E. (2020, July 11). Human Intelligence and General Collective Intelligence as Phase Changes in Animal Intelligence. https://doi.org/10.31234/osf.io/dr8qn 
[7] Williams, A. E., Defining a Continuum from Individual, to Swarm, to Collective Intelligence, to General Collective Intelligence, International Journal of Collaborative Intelligence, in print (2021) [8] Herbert S. Green (1965). Matrix mechanics (P. Noordhoff Ltd, Groningen, Netherlands) ASIN : B0006BMIP8.

[9] Weinberg, S. (2002) [1995], Foundations, The Quantum Theory of Fields, 1, Cambridge: Cambridge University Press, ISBN 978-0-521-55001-7

[10] Schrödinger, E. (1926). "An Undulatory Theory of the Mechanics of Atoms and Molecules" (PDF). Physical Review. 28 (6): 1049-1070.

[11] Lorenz, E. N. (1963). Deterministic nonperiodic flow. Journal of atmospheric sciences,20(2):130141.

[12] Williams A.E. (2020) A Model for Artificial General Intelligence. In: Goertzel B., Panov A., Potapov A., Yampolskiy R. (eds) Artificial General Intelligence. AGI 2020. Lecture Notes in Computer Science, vol 12177. Springer, Cham. https://doi.org/10.1007/978-3-030-52152-3 38

[13] Williams, A.E. Are wicked problems a lack of general collective intelligence?. AI \& Soc (2021). https://doi.org/10.1007/s00146-021-01297-8 